\title{
Modal Spectroscopy of Optoexcited Vibrations of a Micron-Scale On-Chip Resonator at Greater than $1 \mathrm{GHz}$ Frequency
}

\author{
Tal Carmon and Kerry J. Vahala \\ California Institute of Technology, 1200 E. California Boulevard, Pasadena, California 91125 USA \\ (Received 5 July 2006; revised manuscript received 23 October 2006; published 20 March 2007)
}

\begin{abstract}
We analyze experimentally and theoretically $>1 \mathrm{GHz}$ optoexcited mechanical vibration in an on-chip micron-scaled sphere. Different eigen-mechanical modes are excited upon demand by the centrifugal radiation pressure of the optical whispering-gallery-mode, enabling an optomechanical modal spectroscopy investigation of many vibrational modes. Spectral analysis of the light emitted from the device enables deduction of its natural vibrational modes in analogy with spectroscopy of a molecule's vibrational levels, and its eccentricity perturbation is shown to induce spectral splitting.
\end{abstract}

The acoustical whispering-gallery mode, first described by Rayleigh [1], has an optical analogue in microspheres that has attracted the attention of many researchers owing to its spatial confinement and low optical dissipation [24]. The coexistence of optical- and mechanical-modes in such devices enables radiation pressure to couple the mechanical and optical-degrees of freedom [5-9], with suggested applications to both classical and quantum phenomena [6,10-15]. Generally, in quantum optomechanical applications, it is preferable for the mechanicaloscillation quanta, $\hbar \Omega$, to be as large as possible when compared with thermal energy, $K_{b} T$.

Here, we optoexcite vibrations in an on-chip micronscaled sphere at microwave rates $(>1 \mathrm{GHz})$ for the first time. Selection of the required single, mechanical mode is accomplished here by control of the photon lifetime and enables optomechanical modal spectroscopy (OMMS) of the natural vibrations of the structure.

Optomechanical oscillation has been demonstrated previously at radio-frequency rates using toroidal resonators [7-9]. Here, we use spherical resonators as a means to boost the mechanical eigen frequencies into the microwave-rate regime. Along these lines, on-chip spherical structures are stiffer and can be fabricated to smaller sizes than microtoroids, enabling higher mechanical eigen frequencies. We demonstrate experimentally an on-chip spheroidal resonator oscillating regeneratively at a single frequency higher than $1 \mathrm{GHz}$.

The micron-scaled silica sphere made on a silicon chip (in analogy to the toroid fabrication process described in Ref. [16]) is formed by reflow of a silica disk. Continuouswave $[\mathrm{cw}]$ optical power is evanescently coupled into the sphere by means of a tapered fiber [17,18] [Fig. 1(b)]. It is necessary here that the coupler will not touch the cavity as touching can quench mechanical vibrations. When the input optical wavelength is tuned to one of the optical resonances of the sphere, optical energy starts to build up in the cavity and centrifugally pushes its walls to inflate. Inflation, in return, takes the sphere out of resonance with the optical pump, and the light leakage through the taper results in deflation. Under conditions where the pump wave is slightly blue detuned relative to the resonance, this process results in regenerative mechanical oscillation [79]. The cycling of optical power to and from the resonator

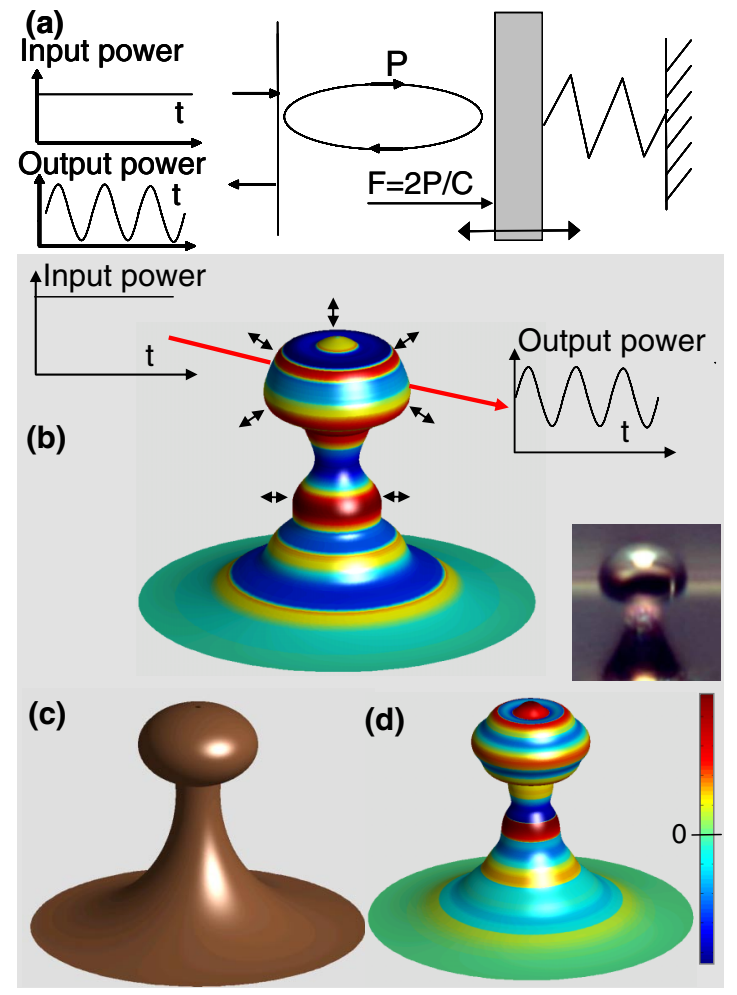

FIG. 1 (color online). (a) Schematics of the radiation-pressure instability in the context of Fabry-Perot resonator. (b) Experimental setup: continuous-wave optical input is evanescently coupled via a tapered fiber to a whispering-gallery-mode cavity. One of the natural mechanical modes of the structure is optoexcited by the centrifugal radiation pressure and modulates the optical output in return. Inset shows a micrograph of the whispering-gallery-mode cavity. (c) Rendering of the cavity at mechanical equilibrium. (d) Rendering of the calculated $538 \mathrm{MHz}$ mode. The calculated deflection is exaggerated in the rendering. 
creates modulation of the transmitted power at the mechanical-oscillation frequency. By monitoring this modulation using a photodetector, the eigen frequency, threshold-power, and even the amplitude of the mechanical oscillation can be easily measured. For a more rigorous explanation, the reader can refer to the dynamical equations describing this system [7-9] as well as to their analytical solution [19].

Schematics of the radiation-pressure instability in the context of Fabry-Perot resonator are provided in Fig. 1(a). Moving to the spherical geometry, the experimental setup with a micrograph of the cavity is given in Fig. 1(b). In Fig. 1(c), the cavity at mechanical equilibrium is shown, and its $538 \mathrm{MHz}$ mechanical mode is rendered in Fig. 1(d). Figures 2(a)-2(e) present four columns of information corresponding to excitation of increasingly higherfrequency mechanical eigen modes of the microsphere. The first column presents the measured spectrum of detected photocurrent. The fundamental frequency component in each spectrum gives the eigen frequency of the mechanical motion while the higher harmonics of this component result from the highly nonlinear nature of the "transfer function" associated with modulation of the optical pump wave by the vibrating optical microsphere resonator. The second column gives the measured timedomain version of the detected photocurrent. In the third

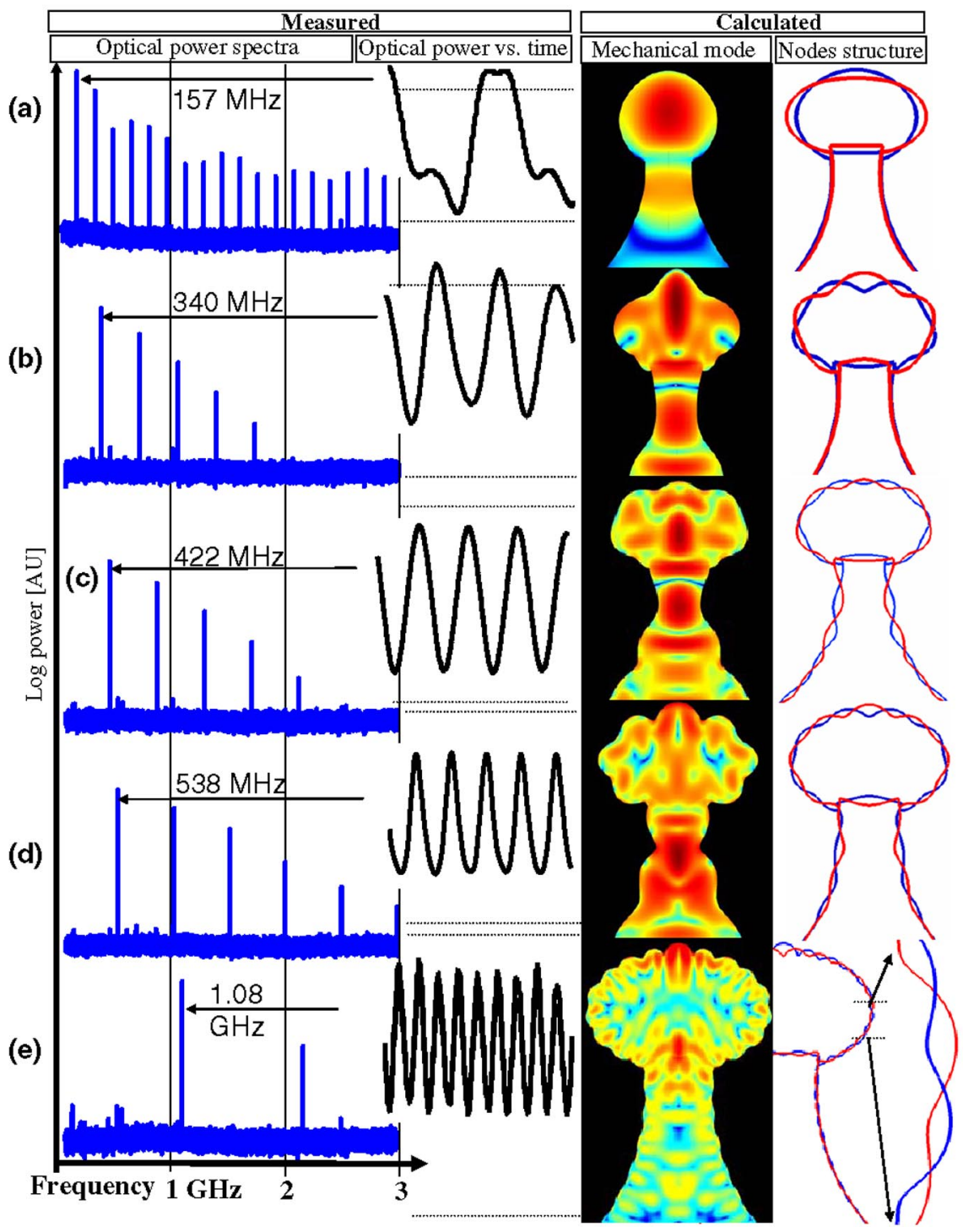

FIG. 2 (color online). Column 1 shows the electrical spectrum of photocurrent upon detection of continuous pump wave modulated by the vibrating microsphere. Column 2 shows the same photocurrent signal except measured in the time domain (horizontal dotted lines in column 2a stands for transmission of 0 and 1). The experimental data is taken at a pump power which is 10 times higher than that of the vibration threshold power. The corresponding calculated mechanical modes are presented in column 3 . Also calculated is the nodes geometry in column 4 wherein the lines represent the calculated boundaries at $\pi / 2$ and $-\pi / 2$. The calculated deflection is exaggerated in the plots. Movies of the vibrational modes are available in Ref. [25]. 
and fourth columns, the calculated strain field and nodes structure of the corresponding mechanical oscillation are presented. The accuracy of the mode solver was verified by comparing its output with the analytical solutions for spheres and cylinders [15].

The first optoexcited natural vibration is measured [Fig. 2(a)] to oscillate at $157 \mathrm{MHz}$. The frequency of the calculated mechanical mode deviates only $1 \%$ from this measured frequency (deviation was smaller than $2.5 \%$ for the other modes). It is visible from the plotted calculation [Fig. 2(a) fourth column] that the shape of the spheroid changes between oblate and prolate while it vibrates. As for other technical details, the optical quality factor of the spherical cavity is $1.7 \times 10^{6}$ as measured by scanning the optical transmission spectrum and measuring the FWHM of the linewidth. Optical pumping was performed at $1555 \mathrm{~nm}$. Oscillation of this fundamental mode starts at $6 \mathrm{~mW}$ of input power (i.e., the threshold power), reflecting a mechanical $Q$ of 142 .

In order to select higher natural frequencies, the lifetime of the optical-cavity photons is shortened to be comparable with the mechanical-vibration period [7-9,19,20]. Lifetime shortening is done here by changing the coupling gap between the tapered fiber and the spherical cavity [21]. This distance for the modes in Fig. 2 is 1500, 1000, 900, 800 , and $200 \mathrm{~nm}$; the narrower coupling gaps improve field coupling and thereby lower the photon lifetime. One acoustical mode was observed to oscillate at each coupling gap setting. As is apparent from Fig. 2(e), this couplinggap degree of freedom allows excitation and selection of mechanical modes with eigen frequencies as high as $1.08 \mathrm{GHz}$

Concerning the $1.08 \mathrm{GHz}$ eigenmode, it contains many nodes along the sphere latitude as calculated in column 4 of Fig. 2(e). Also apparent from the nodes structure is that the mode extends beyond the silica sphere and well into the silicon support structure. This fact is no doubt in large part responsible for the low mechanical $Q$ factors measured in this system. As for additional technical details concerning all of the eigen modes measured in Fig. 2(b)-2(e), the corresponding threshold power was $24,31,74$, and $98 \mathrm{~mW}$.

In Fig. 3, the fundamental spectral peak from Fig. 2(a) is scanned at higher resolution in the temporal-spectral domain (3a) and at high resolution in the spectral domain (3b). The instrument resolution setting in Fig. 3(b) is $50 \mathrm{~Hz}$, and by using a fit to the spectrum, we infer a FWHM Lorentzian linewidth of $300 \mathrm{~Hz}$, which is 6 orders of magnitude smaller than the typical vibrational frequency, giving a measure of the ability to distinguish between different OMMS signatures. In fact, this frequency/width ratio is so high that distinct cavities tested were easily distinguished using only their OMMS signature. This linewidth is also narrow enough to distinguish spectral-line splitting caused by perturbation. In particular, by introducing a slight eccentricity into the toroidal cavity, it was possible to induce a slight anisotropy in the silica toroid. Such a perturbation will result in anisotropy of the excitation radiation force and enable excitation of higherorder vibrational modes. These standing-wave modes occur in doublets oriented with different rotation that are slightly split in their eigen frequencies due to the presence of the azimuthal perturbation. Such a measured doublet pair is observed using OMMS in Fig. 4(a). Calculations presented in Fig. 4(b) show the vibrational modes corresponding to the observed spectral split pair. It is worth commenting that perturbation-induced degeneracy splitting such as this is analogous to Stark splitting, for example, induced by an anisotropic field.

To conclude, $1.08 \mathrm{GHz}$ regenerative oscillation of an optomechanical resonator is demonstrated here. Modal spectroscopy is demonstrated via agreement between the many calculated and measured spectral lines (Fig. 2), including high resolution observation of a perturbationinduced splitting in vibrational modes (Fig. 4).

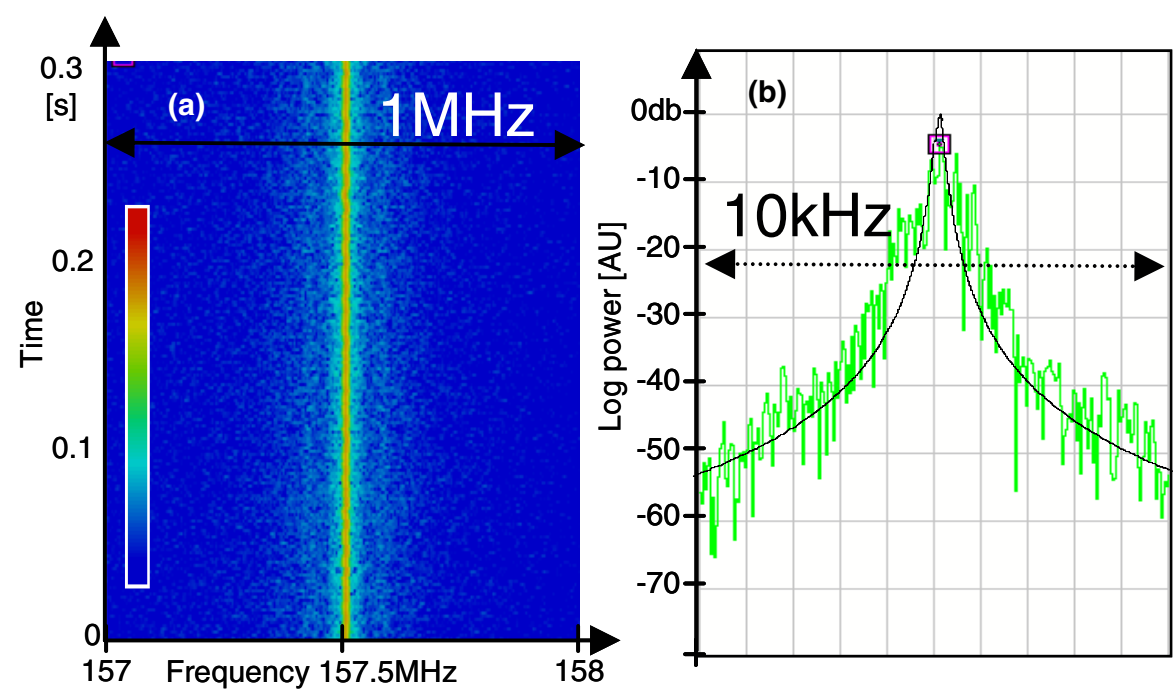

FIG. 3 (color online). Higher resolution spectral scans (over two scan-width spans 3a,b) of the fundamental spectral component in Fig. 2(a). (3a) contains a temporal-spectral scan and ( $3 b)$ contains a conventional spectral scan. Using the Lorentzian fit in figure $3 b$, we infer a FWHM linewidth for the oscillation peak (and hence the mechanical mode) of $300 \mathrm{~Hz}$. The data are collected for excitation at $10 X$ the threshold power. 

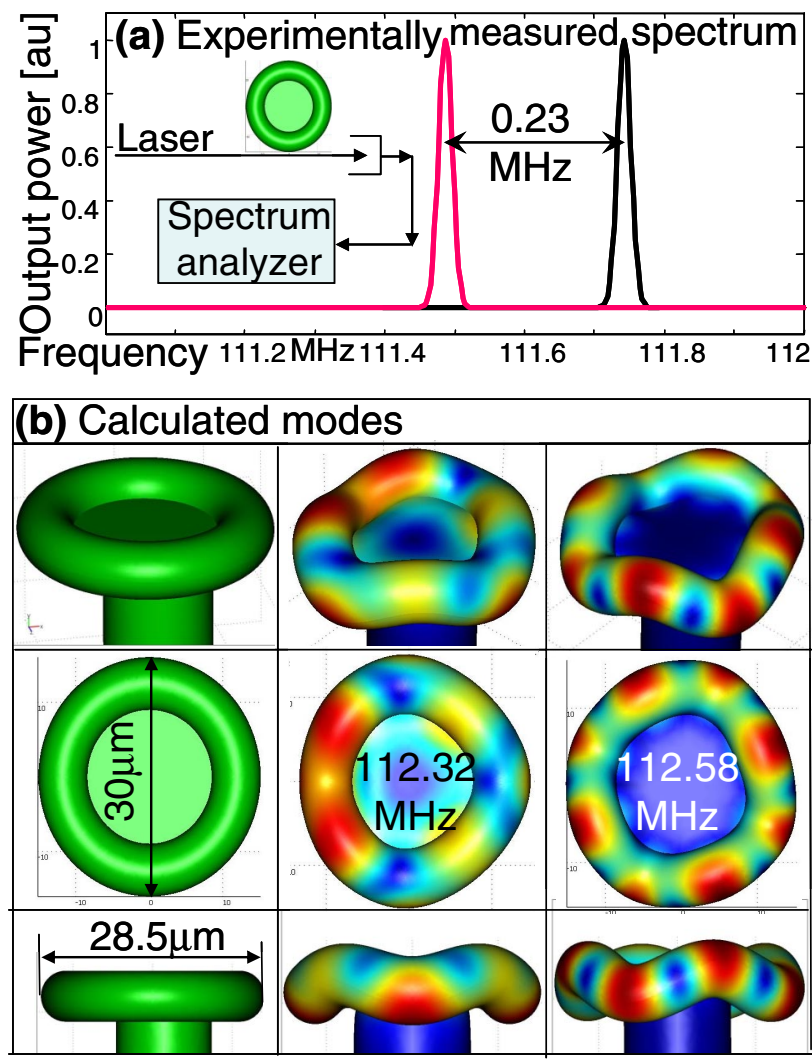

FIG. 4 (color online). (a) Experimental spectrum of an anisotropic toroidal cavity reveals spectral-line splitting (light and dark lines). (b) Calculation shows the corresponding degenerate modes oriented with different rotation. Deformation is exaggerated in figures.

In the future, going to a cryogenic and/or a vacuum environment as well as reducing the pillar diameter is expected to increase mechanical $Q$ and hence lower pump thresholds for all modes and allow higher oscillation frequencies. Recent reports on optomechanical effects in photonic crystal fiber $[23,24]$ and the availability of microlevers cavities [22] suggest that OMMS in many more platforms is possible. Finally, from a wider point of view, the work presented here shares some similarities to vibrational spectroscopy of a molecule in which pumped optical modes play the role of electronic states, and coupling of these states to vibrational degrees of freedom is apparent in scanned transmission spectra.

We acknowledge support from the Caltech Lee Center and DARPA. Carmon acknowledges support from the Center for the Physics of Information and discussions with Hongzing Tang.
[1] L. Rayleigh, Philos. Mag. 20, 1001 (1910).

[2] C. G. Garrett, W. Kaiser, and W. L. Bond, Phys. Rev. 124, 1807 (1961)

[3] K. J. Vahala, Nature (London) 424, 839 (2003).

[4] V.S. Ilchenko and A.B. Matsko, IEEE J. Sel. Top. Quantum Electron. 12, 15 (2006).

[5] A. Dorsel, J.D. McCullen, P. Meystre, E. Vignes, and H. Walther, Phys. Rev. Lett. 51, 1550 (1983).

[6] V. B. Braginsky, S. E. Strigin, and S. P. Vyatchanin, Phys. Lett. A 287, 331 (2001).

[7] T. Carmon, H. Rokhsari, L. Yang, T. J. Kippenberg, and K. J. Vahala, Phys. Rev. Lett. 94, 223902 (2005).

[8] H. Rokhsari, T. J. Kippenberg, T. Carmon, and K.J. Vahala, Opt. Express 13, 5293 (2005).

[9] T. J. Kippenberg, H. Rokhsari, T. Carmon, A. Scherer, and K. J. Vahala, Phys. Rev. Lett. 95, 033901 (2005).

[10] L. Hilico, J. M. Courty, C. Fabre, E. Giacobino, I. Abram, and J. L. Oudar, Appl. Phys. B 55, 202 (1992).

[11] V. Giovannetti, S. Mancini, and P. Tombesi, Europhys. Lett. 54, 559 (2001).

[12] W. Marshall, C. Simon, R. Penrose, and D. Bouwmeester, Phys. Rev. Lett. 91, 130401 (2003).

[13] S. Mancini, V. Giovannetti, D. Vitali, and P. Tombesi, Phys. Rev. Lett. 12040188 (2002).

[14] S. Mancini, D. Vitali, and P. Tombesi, Phys. Rev. Lett. 90, 137901 (2003).

[15] D. Vitali, S. Mancini, L. Ribichini, and P. Tombesi, J. Opt. Soc. Am. B 20, 1054 (2003).

[16] D. K. Armani, T. J. Kippenberg, S. M. Spillane, and K. J. Vahala, Nature (London) 421, 925 (2003).

[17] J. C. Knight, G. Cheung, F. Jacques, and T. A. Birks, Opt. Lett. 22, 1129 (1997).

[18] S. M. Spillane, T. J. Kippenberg, O. J. Painter, and K. J. Vahala, Phys. Rev. Lett. 91, 043902 (2003).

[19] F. Marquardt, J. G. E. Harris, and S. M. Girvin, Phys. Rev. Lett. 96, 103901 (2006).

[20] H. Rokhsari, I. J. Kippenberg, T. Carmon, and K. J. Vahala, IEEE J. Sel. Top. Quantum Electron. 12, 96 (2006).

[21] M. L. Gorodetsky and V.S. Ilchenko, J. Opt. Soc. Am. B 16, 147 (1999).

[22] C. H. Metzger, and K. Karrai, Nature (London) 432, 1002 (2004).

[23] P. Dainese, P. S. J. Russell, N. Joly, J. C. Knight, G. S. Wiederhecker, H. L. Fragnito, V. Laude, and A. Khelif, Nature Phys. 2, 388 (2006).

[24] P. Dainese, P. S. J. Russell, G. S. Wiederhecker, N. Joly, H. L. Fragnito, V. Laude, and A. Khelif, Opt. Express 14, 4141 (2006).

[25] See EPAPS Document No. E-PRLTAO-98-049709. The movies can be viewed at http://ftp.aip.org/epaps/phys_ rev_lett/E-PRLTAO-98-049709/. For more information on EPAPS, see http://www.aip.org/pubservs/epaps.html. 